\title{
The Impact of Frequency and Tone of Parent-Youth Communication on Type 1 Diabetes Management
}

\author{
Mark D. DeBoer · Rupa Valdez • Daniel R. Chernavvsky • Monica Grover • \\ Christine Burt Solorzano $\cdot$ Kirabo Herbert $\cdot$ Stephen Patek
}

Received: February 27, 2017 / Published online: April 12, 2017

(C) The Author(s) 2017. This article is an open access publication

\begin{abstract}
Background: The purpose of this study is to assess the impact of frequency and tone of parent-youth communication on glycemic control as measured by the Family Communication Inventory (FCI). Adolescence provides a unique set of diabetes management challenges,
\end{abstract}

Enhanced Content To view enhanced content for this article go to http://www.medengine.com/Redeem/ 8C08F0601556E12B.

Electronic supplementary material The online version of this article (doi:10.1007/s13300-017-0259-2) contains supplementary material, which is available to authorized users.

M. D. DeBoer $(\bowtie) \cdot$ M. Grover · C. Burt Solorzano · K. Herbert

Department of Pediatrics, University of Virginia Health System, Charlottesville, VA, USA

e-mail: deboer@virginia.edu

R. Valdez

Department of Public Health Sciences, University of Virginia School of Medicine, Charlottesville, VA 22908, USA

D. R. Chernavvsky

Department of Psychiatry and Neurobehavioral

Sciences, University of Virginia School of Medicine,

Charlottesville, VA 22908, USA

\section{S. Patek}

Department of Systems and Information Engineering, University of Virginia School of Medicine, Charlottesville, VA 22908, USA including suboptimal glycemic control. Continued parental involvement in diabetes management is associated with improved HbA1c outcomes; however, diabetes-related conflict within the family can have adverse effects. Although it is clear that communication plays an important role in diabetes outcomes, the specific impact of frequency and tone of such communication is largely understudied.

Methods: A total of 110 youths with type 1 diabetes and their parents completed questionnaires assessing diabetes-related adherence, family conflict, and family communication (i.e., frequency and tone) during a routine clinic visit. Routine testing of HbA1c was performed.

Results: Youth- and parent-reported frequency of communication were unrelated to HbA1c. Instead, greater discrepancies between parents and children on reported frequency of communication (most commonly parents reporting frequent and youth reporting less frequent communication) corresponded with poorer glycemic control and increased family conflict. More positive tone of communication as rated by youth was associated with lower HbA1c.

Conclusions: Diabetes-related communication is more complex than conveyed simply by how often children and their parents communicate. Tone of communication and discrepancies in a family's perception of the frequency of communication were better than frequency as predictors of glycemic control. The FCI appears to capture the frequency and tone of 
diabetes-related communication, though larger-scale studies are warranted to inform future use of this scale.

Keywords: Adolescent;

Communication; Diabetes mellitus; Family conflict; Parents; Surveys and questionnaires

\section{INTRODUCTION}

Adolescents with type 1 diabetes (T1D) face a myriad of obstacles to maintaining adequate blood glucose (BG) control, resulting in HbA1c levels that are the highest of any age group [1]. Navigating the division of responsibility of diabetes management tasks between parents and youth is a particularly salient challenge for this population. Although it is recognized that adolescents need to engage in some degree of developmentally appropriate diabetes self-management behaviors to prepare them for life apart from their parents [2-4], it is well established that they commonly have difficulty carrying out some of these tasks [5-9]. In addition to the inherent challenges of adolescence, changes that occur within parent-youth relationships and communication patterns during this developmental transition may also have an impact on diabetes management and glycemic outcome. Continued parental involvement in adolescents' diabetes management is recommended and has been associated with better outcomes (i.e., lower HbA1c) [10-14]. The quality and degree of parental monitoring may also have an impact on a youth's adherence and subsequent glycemic control, with some evidence suggesting that parental monitoring is associated with improved self-efficacy, adherence, and HbA1c in adolescents [15]. However, perceived parental over-involvement has been associated with poorer glycemic control and more episodes of diabetic ketoacidosis [16].

Although family functioning [17] and co-regulation of care between parents and children $[12,13]$ appear to be important predictors of diabetes control, the role of diabetes-related communication in diabetes management is not fully understood. Most research efforts have focused on the quality of family communication, demonstrating that increased diabetes-related family conflict $[7,18-20]$, parental negativity $[17,21]$, and parental criticism $[17,22]$ are related to suboptimal glycemic control in youth, while perceived parental warmth, effective conflict resolution, and parental positive reinforcement are associated with better glycemic control [23]. Negative family communication, specifically diabetes-related conflict, also has a demonstrated negative impact on both general and diabetes-specific quality of life for youth with T1D [24, 25]. In fact, Laffel and colleagues found that diabetes-related family conflict was the only significant predictor of quality of life after controlling for demographic variables such as age, gender, and duration of diabetes, as well as HbA1c and parental involvement in insulin administration [24].

While previous studies have investigated the relationship between degree of conflict and T1D-related outcomes, they have not examined the role of family communication frequency or valence (positive to negative tone). Valence has the potential to offer additional insights into the impact of family communication on diabetes management because negative tone can encompass more subtle interactions in parent-youth dyads beyond explicit conflict (e.g., sarcasm). Moreover, discrepancies between parent and youth perceptions of the overall frequency and valence of communication may also play an important role in promoting adaptive diabetes care. Previous research has demonstrated that discrepancies in perceptions of family members' responsibility for diabetes care are predictive of poorer metabolic outcomes [26].

The goal of this study was to investigate the role of parent- and youth-reported frequency and valence of communication about T1D in overall glycemic control. To achieve this goal, we developed and tested a T1D communication questionnaire, the Family Communication Inventory (FCI). Our primary hypothesis was that higher frequency and more positive tone of communication between youth and their parents would be associated with lower HbA1c values. Our secondary hypothesis was that greater discrepancies in parent-youth 
perceptions regarding frequency and tone of communication would be associated with suboptimal metabolic control as determined by higher HbA1c values and poorer diabetes management behaviors.

\section{METHODS}

This study was approved by the University of Virginia (UVA) Social and Behavioral Sciences Institutional Review Board. All procedures followed were in accordance with the ethical standards of the responsible committee on human experimentation (institutional and national) and with the Helsinki Declaration of 1964, as revised in 2013. Informed consent was obtained from all patients for being included in the study. Eligible individuals were youth between the ages of 12 and 18 years and their accompanying parents who were at a clinic visit to receive care at the UVA Diabetes Clinic for T1D. Participants and their accompanying parent were invited to participate in the study by clinic nursing staff at their routine diabetes clinic visit. If two parents were present at the visit, the parent who assumes primary responsibility of the youth's diabetes care was asked to participate. Participants were informed that their responses were completely confidential and that their participation or lack thereof would not affect their clinical care in any way. Upon informed consent and assent, participants and their parents were provided with parent and youth self-report questionnaires to complete independently at the clinic visit. All completed questionnaires were collected by the front desk staff at the end of the clinic visit. The adolescent received a US\$20 gift card for participation.

\section{Measures}

\section{Glycemic control}

Glycated hemoglobin (HbA1c) was used to provide an estimate of the youths' glycemic control for the preceding 2-3 months. Clinical nursing staff collected blood samples via finger stick during the clinic visit, which were analyzed using a DCA Vantage Analyzer ${ }^{\mathrm{TM}}$ (Siemens AG, Munich, Germany).

\section{Questionnaires}

Diabetes Family Conflict Scale-Revised (DFCS) The revised DCFS was used to assess diabetes-specific family conflict over the past month [19]. It consists of 19 items scored on a 3 -point Likert scale $(1=$ never argue, 2 = sometimes argue, 3 = always argue), summed to yield a total score ranging between 19 (no conflict) and 57 (high conflict). The revised DCFS previously demonstrated adequate internal consistency for both youth and caregiver reports ( $\alpha=0.85, \alpha=0.81$ respectively) and had high internal consistency with the present sample (youth $\alpha=0.91$, parent $\alpha=0.92$ ) [19].

Child Self-Management Scale (CSM) The CSM was developed for the present study (Supplementary Table 1) to measure youth adherence to diabetes management tasks over the past 2 weeks. It is scored as the mean of six items that assess the frequency of missing specific required self-management tasks (e.g., "Missed taking insulin for a meal"), which are responded to on a 5-point Likert scale $(0=$ Never, $4=$ seven or more times). Higher scores indicate more difficulty with adherence to diabetes management tasks.

Family Communication Inventory (FCI) The FCI is a nine-item measure created for this study to assess parent-youth communication, including frequency and tone, related to the youth's diabetes management (Table 1). Items were generated by a pediatric endocrinologist and human factors specialist on the basis of clinical and research-related experience interacting with families. Parents and youth were instructed to rate how often they communicated about specific diabetes-related tasks within the past week on a 5-point Likert scale ranging from 0 (never) to 4 (multiple times daily). For each task, they were also asked to rate the tone of the communication on a 5-point Likert scale ranging from 0 (very negative) to 4 (very positive). If a participant indicated that a diabetes-related task was never discussed, there was an option to indicate that the tone of the communication was "not applicable." Higher item mean scores on frequency of communication (FCom) indicate more frequent 
Table 1 Family communication scales: frequency and tone of communication

\begin{tabular}{|c|c|c|c|c|c|c|c|}
\hline \multirow[t]{2}{*}{ Item } & \multicolumn{3}{|l|}{ Youth } & \multicolumn{3}{|l|}{ Parent } & \multirow{2}{*}{$\begin{array}{l}\text { Correlation } \\
\text { (youth to parent) } \\
\text { Spearman's rho }\end{array}$} \\
\hline & Mean & SD & $\begin{array}{l}\text { Correlation } \\
\text { to tone }\end{array}$ & Mean & SD & $\begin{array}{l}\text { Correlation } \\
\text { to tone }\end{array}$ & \\
\hline $\begin{array}{l}\text { FCom 1: discussed insulin dosing of a } \\
\text { particular meal }\end{array}$ & 2.67 & 1.36 & $0.228^{*}$ & 2.89 & 1.31 & $0.246^{*}$ & $0.599^{* *}$ \\
\hline TCom $1 \neq \phi$ & 3.83 & 0.70 & - & 3.68 & 0.79 & - & $0.538^{* *}$ \\
\hline $\begin{array}{l}\text { FCom 2: discussed insulin dosing of a } \\
\text { particular high blood sugar }\end{array}$ & 2.29 & 1.05 & 0.092 & 2.29 & 0.91 & 0.038 & $0.480^{* *}$ \\
\hline TCom $2 \neq \phi$ & 3.68 & 0.79 & - & 3.54 & 0.86 & - & $0.625^{* *}$ \\
\hline $\begin{array}{l}\text { Fcom 3: discussed treatment of a } \\
\text { particular low blood sugar } \phi(-)\end{array}$ & 2.06 & 0.95 & 0.150 & 2.00 & 0.87 & 0.183 & $0.528^{* *}$ \\
\hline TCom 3 & 3.85 & 0.76 & - & 3.89 & 0.69 & - & $0.423^{* *}$ \\
\hline $\begin{array}{l}\text { FCom 4: discussed missed insulin boluses } \\
\quad \neq\end{array}$ & 1.50 & 0.84 & $-0.471^{* *}$ & 1.47 & 0.82 & $-0.431^{* *}$ & $0.353^{* *}$ \\
\hline TCom 4 & 3.55 & 0.92 & - & 3.34 & 1.00 & - & $0.551^{* *}$ \\
\hline $\begin{array}{l}\text { FCom 5: discussed overall blood sugar } \\
\text { control }\end{array}$ & 2.80 & 1.18 & 0.181 & 2.89 & 1.12 & 0.151 & $0.526^{* *}$ \\
\hline TCom $5 \neq \phi$ & 3.61 & 0.87 & - & 3.53 & 0.83 & - & $0.616^{* *}$ \\
\hline $\begin{array}{l}\text { FCom 6: discussed frequency/number of } \\
\text { low blood sugars } \neq(-) \phi(-)\end{array}$ & 1.96 & 0.98 & 0.178 & 1.93 & 0.98 & 0.068 & $0.555^{* *}$ \\
\hline TCom $6 \neq$ & 3.68 & 0.74 & - & 3.78 & 0.66 & - & $0.481^{* *}$ \\
\hline $\begin{array}{l}\text { FCom 7: discussed frequency/number of } \\
\text { high blood sugars } \neq\end{array}$ & 2.51 & 1.05 & -0.084 & 2.42 & 0.91 & -0.002 & $0.393^{* *}$ \\
\hline TCom $7 \neq \phi$ & 3.50 & 0.88 & - & 3.47 & 0.93 & - & $0.704^{* *}$ \\
\hline $\begin{array}{l}\text { FCom 8: discussed adjustment of } \\
\text { insulin/carb ratio }\end{array}$ & 1.74 & 0.99 & -0.105 & 1.75 & 1.00 & 0.124 & $0.578^{* *}$ \\
\hline TCom $8 \neq$ & 3.82 & 0.72 & - & 3.75 & 0.72 & - & $0.699^{* *}$ \\
\hline $\begin{array}{l}\text { Fcom 9: discussed adjustment of Lantus/ } \\
\text { basal rates }\end{array}$ & 1.57 & 0.83 & 0.079 & 1.51 & 0.73 & 0.066 & $0.502^{* *}$ \\
\hline TCom $9 \neq$ & 3.84 & 0.78 & - & 3.88 & 0.72 & - & $0.662^{* *}$ \\
\hline
\end{tabular}


Table 1 continued

\begin{tabular}{|c|c|c|c|c|c|c|c|}
\hline \multirow[t]{2}{*}{ Item } & \multicolumn{3}{|l|}{ Youth } & \multicolumn{3}{|c|}{ Parent } & \multirow{2}{*}{$\begin{array}{l}\text { Correlation } \\
\text { (youth to parent) } \\
\text { Spearman's rho }\end{array}$} \\
\hline & Mean & SD & $\begin{array}{l}\text { Correlation } \\
\text { to tone }\end{array}$ & Mean & SD & $\begin{array}{l}\text { Correlation } \\
\text { to tone }\end{array}$ & \\
\hline & & & & & & & Pearson's $R$ \\
\hline FCom overall & 2.13 & 0.69 & 0.077 & 2.14 & 0.64 & 0.077 & $0.604^{* *}$ \\
\hline TCom overall & 3.73 & 0.63 & - & 3.71 & 0.67 & - & $0.831^{* *}$ \\
\hline
\end{tabular}

* Correlation is significant at $p<0.05$

** Correlation is significant at $p<0.01$

₹Youth response was significantly related to HbAlc $(p<0.05)$, controlling for youth age, gender, and duration of diabetes; $(-)$ indicates that the increased frequency was associated with lower HbAlc

$\phi$ Parent response was significantly related to $\mathrm{HbAlc}(p<0.05)$, controlling for youth age, gender, and duration of diabetes; $(-)$ indicates that increased frequency was associated with lower HbAlc

communication, and higher item mean scores on tone of communication (TCom) indicate more positive tone.

\section{Discrepancy Data}

Evidence suggests that discrepancies in perspectives on diabetes care between children and parents can negatively impact overall diabetes control [26]. To test whether this association is also the case for frequency and tone of communication, we developed a measure to calculate the mean difference in response to FCI items between each youth-parent dyad. The measure is akin to a standard deviation and is given by Eq. 1 where $x$ is each item (e.g., FCom 4 ) and $n$ is the total number of items on the scale:

Standard deviation $\mathrm{Agreement}_{\text {}}$

$$
=\sqrt{\frac{\sum_{x=1}^{n}\left(\text { Parent score }_{\text {item } x}-\text { Youth score }_{\text {item } x}\right)^{2}}{N \text { items }}}
$$

Disagreement score is calculated by taking the square root of the sum of squared differences of all scale items divided by the total number of items. Disagreement scores give the mean difference of reported frequency and tone between parents and youth, or the magnitude of discrepancy. A disadvantage of this measure is that it removes the direction of differences (i.e., whether youth believe there is more frequent communication than parents). Therefore, we separately summed the total number of items where parents or youth endorsed higher values on the FCI scales to quantify the direction of discrepancies in communication.

\section{RESULTS}

\section{Participant Characteristics}

We assessed a total of 110 participants with T1D (50\% female), with a mean age of 14.5 years (range 12-18). Participants' medical characteristics included a mean duration of diabetes of 6.6 years (range $0.04-15$ ) and mean HbA1c was $8.7 \% \quad(71.6 \mathrm{mmol} / \mathrm{mol}) \quad$ (range $5.6-14.0 \%$; $37.7-129.5 \mathrm{mmol} / \mathrm{mol}$-above the level of $7.5 \%(58.5 \mathrm{mmol} / \mathrm{mol})$ recommended by the American Diabetes Association for this age range [27].

\section{Frequency of Communication (FCom)}

\section{Scale Characteristics}

Table 1 shows the mean, standard deviation, and correlations between parents and youth for each FCom item, while Supplementary Table 2 provides the number or responses to each item. Averaging across items, parent $(M=2.13 \pm 0.69)$ and youth $(M=2.14 \pm 0.64)$ scores correlated highly on the frequency of 
Table 2 Factor loadings for parents and youth on the frequency of communication (FCom) items

\begin{tabular}{lll}
\hline Item & Youth factor loading & Parent factor loading \\
\hline FCom 1: discussed insulin dosing of a particular meal & 0.464 & 0.339 \\
FCom 2: discussed insulin dosing of a particular high blood sugar & 0.775 & 0.782 \\
FCom 3: discussed treatment of a particular low blood sugar & 0.828 & 0.839 \\
FCom 4: discussed missed insulin boluses & 0.802 & 0.750 \\
FCom 5: discussed overall blood sugar control & 0.451 & 0.373 \\
FCom 6: discussed frequency/number of low blood sugars & 0.844 & 0.878 \\
FCom 7: discussed frequency/number of high blood sugars & 0.688 & 0.777 \\
FCom 8: discussed adjustment of insulin/carb ratio & 0.900 & 0.842 \\
FCom 9: discussed adjustment of Lantus/basal rates & 0.904 & 0.855 \\
\hline
\end{tabular}

diabetes communication $(r=0.60, \quad n=109$, $\left.p<0.0005, r^{2}=36.5 \%\right)$. Cronbach's alphas for youth $(\alpha=0.84)$ and parents $(\alpha=0.81)$ were good for the nine-item scale. Exploratory factor analysis (EFA) conducted with principal axis factoring revealed that all FCom items loaded onto a single factor at Eigen values greater than 0.30 for both parents and youth respondents (see Table 2), while a two-factor solution yielded cross loading on multiple items.

On average, parents and youth disagreed by less than 1 point per FCom item $(M=0.85 \pm 0.63, \quad$ range $=0.0-2.67) . \quad$ Overall, these discrepancies were balanced, with youth and parents reporting more communication on an average of 1.89 items and 1.86 items, respectively, $t(100)=0.094, p=0.925$.

\section{Relationship to $\mathrm{HbA1c}$}

In separate linear regression models, neither youth nor parent mean FCom scores significantly predicted HbA1c after controlling for youth age, duration of diabetes, and gender. However, FCom disagreement score was positively associated with HbA1c (standardized $\beta=0.22, t(99)=2.27, p=0.026)$, suggesting that more youth-parent disagreement about frequency of communication corresponded with higher HbA1c scores.

Table 3 Pearson's correlations between frequency/tone of communication, the family conflict scale, and the child self-management scale

\begin{tabular}{lccccc}
\hline & Parent FCS & Youth FCS & Parent CSM & Youth CSM & CSM disagreement \\
\hline Parent FCom & -0.047 & -0.063 & -0.079 & $-0.177^{*}$ & 0.005 \\
Youth FCom & -0.061 & 0.076 & 0.013 & 0.010 & 0.128 \\
Frequency disagreement & $0.216^{*}$ & $0.209^{*}$ & $0.169^{*}$ & $0.222^{*}$ & $0.428^{* *}$ \\
Parent TCom & $-0.342^{* *}$ & $-0.319^{* *}$ & $-0.352^{* *}$ & $-0.257^{*}$ & $-0.287^{*}$ \\
Youth TCom & $-0.471^{* *}$ & $-0.521^{* *}$ & $-0.388^{* *}$ & $-0.388^{* *}$ & $-0.397^{* *}$ \\
Tone disagreement & $0.267^{*}$ & $0.460^{* *}$ & $0.604^{* *}$ & $0.445^{* *}$ & $0.677^{* *}$ \\
\hline
\end{tabular}

* Correlation is significant at $p<0.05$

** Correlation is significant $p<0.01$ 


\section{Relationship to DFCS}

Table 3 displays Pearson's correlations between youth and parent FCom scores and scores on the DFCS. No significant relationships emerged between mean FCom scores or total conflict scores for parents or youth. However, a positive correlation was found between FCom disagreement scores and parent and youth total conflict scores, suggesting that greater disagreement about frequency of communication was associated with more family conflict as reported by both youth and their parents.

\section{Tone of Communication (TCom)}

\section{Scale Characteristics}

Table 1 shows the mean, standard deviation, and correlation with tone for all respective FCI items, and correlations between parents and youth ratings for each TCom item, while Supplementary Table 2 provides the number or responses to each item. Parent mean TCom scores $(M=3.71 \pm 0.67)$ highly correlated with youth mean scores $(M=3.73 \pm 0.63)(r=0.83$, $\left.n=51, \quad p<0.001, \quad r^{2}=69.1 \%\right)$. Although a single-factor TCom scale appears to have face validity for parents and youth, conducting EFA and Cronbach's alpha analysis was deemed inappropriate because families who did not report communicating about a particular topic were unable to endorse whether any communication on that topic was positive or negative (i.e., there could not have been a particular tone of communication if no communication occurred on that topic in the given time frame). In total 39 youths and 36 parents responded to all nine TCom items, with individual items receiving response rates between $50.0 \%$ and $92.7 \%$ (youth) and 45.5-90.9\% (parents).

To ensure that parent-youth dyad tone scores could be adequately compared, discrepancy scores were only calculated for dyads that responded to at least seven of the same TCom items. For the 48 dyads meeting this criterion, parents and youth disagreed by an average of less than 1 point per TCom item $(M=0.49 \pm 0.39$, range $=0.0-1.27)$.

\section{Relationship to $\mathrm{HbA1c}$}

In a linear regression model, more positive youth TCom scores were associated with lower HbA1c after controlling for youth age, duration of diabetes, and gender (standardized $\beta=-0.45, t(64), p<0.001)$, but no associations were found for TCom parent mean or discrepancy scores. Again, we performed separate regressions to test if individual TCom items significantly predicted $\mathrm{HbA1c}$, after controlling for youth age, duration of diabetes, and gender. All significant youth and parent TCom items indicated that better metabolic control was linked to more positive tone of communication (see Table 1).

\section{Relationship to DFCS}

Table 3 also shows Pearson's correlations between parent/youth DFCS scores and parent/ youth mean TCom scores; there were 107 fully completed parent/youth surveys. Parent TCom scores correlated negatively with both parent and youth conflict scores, suggesting that more positive communication was associated with less perceived family conflict. This relationship was also found between youth TCom scores and parent and youth conflict scores. Finally, TCom disagreement scores were positively related to both parent and youth conflict scores, suggesting that more disagreement about the tone of communication was related to greater perceived family conflict.

\section{Child Self-Management Scale (CSM)}

Child self-management items, mean scores, and correlations between parents and youth are shown in Supplementary Table 1; there were 109 fully completed parent/youth surveys. Cronbach's alphas for youth $(\alpha=0.66)$ and parents $(\alpha=0.70)$ were lower than expected, but similar to previous adherence measures [28]. In separate models controlling for youth age, duration of diabetes, and gender, mean CSM score was positively associated with $\mathrm{HbA1c}$ for both youth (standardized $\beta=0.44$, $t(108)=4.87, p<0.001)$ and parents (standardized $\beta=0.36, t(108)=3.71, p<0.001)$. 
This suggests a relationship between more parent/youth-reported frequency of lapses in diabetes management behaviors and suboptimal youth diabetes control. Greater disagreement between parents and youth on the CSM also corresponded with higher HbA1c (standardized $\beta=0.30, t(106)=3.22, p=0.002)$.

Table 3 shows Pearson's correlations between the CSM and FCom/TCom scores. There was a significant negative association between parent mean FCom scores and youth mean CSM scores, suggesting that more frequent parent reported communication was related to fewer missed diabetes management behaviors. Greater disagreement on the FCom scale positively related to mean parent, mean youth, and disagreement scores on the CSM scale. This suggests that more disagreement between youth and parents about frequency of communication coincided with increased frequency of lapses in diabetes management behaviors, and greater discrepancy between youth and parents about how often lapses occurred. Finally, higher mean parent, mean youth, and disagreement scores on the CSM were associated with more negative tone as reported by parents and youth.

\section{Full Multivariate Model Predicting HbA1c}

As a final exploratory analysis, hierarchical multiple regression was used to test adherence (measured through the CSM) as a potential mediator for the relationship between youth mean TCom and FCom disagreement scores with HbA1c. There was evidence of collinearity between parent- and youth-reported CSM scores; therefore, only youth mean CSM scores were used in the analysis (results were similar using parent mean scores). Entering age, duration of diabetes, gender, and mean youth CSM scores yielded a significant regression model, $F(4,55)=4.98, p=0.002$, which accounted for $26.6 \%$ of the variance in HbA1c. Adding youth TCom and FCom disagreement scores explained an additional $7.2 \%$ of the variance, with this change trending toward significance, $F(2$, $53)=2.90, p=0.064$. In the full multivariate model, FCom disagreement scores were not significantly associated with HbA1c (standardized $\beta=0.090, \quad t \quad(59)=0.72$, $p=0.477$ ), while youth mean CSM (standardized $\beta=0.35, t(59)=2.91, p=0.005)$ and youth mean TCom (standardized $\beta=-0.28$, $t(59)=-2.21, p=0.031)$ scores related significantly to adolescent glycemic control.

\section{DISCUSSION}

This is the first study to examine the frequency and tone of communication between youth and their parents on various diabetes management behaviors. Contrary to the primary hypothesis, no associations were found between overall frequency of communication and glycemic control. Additionally, there were no significant associations between $\mathrm{HbA} 1 \mathrm{c}$ and parent-reported tone of communication or parent-youth disagreement about tone of communication. Instead, results indicated that $\mathrm{HbA1c}$ was significantly related to the disagreement between youth- and parent-reported frequency of communication, with greater disagreement being associated with poorer glycemic control. HbA1c was also significantly associated with tone of communication as reported by the adolescent, where more negative tone correlated with higher HbA1c. These findings are consistent with previous research on diabetes-related family discrepancies in perceptions about responsibility and communication for aspects of diabetes management $[2,16,17,26]$. After including adherence in the regression model, FCom disagreement scores no longer contributed significantly to HbA1c. This finding suggests that discrepancies about frequency of communication may reflect the presence of less than ideal family communication that contribute to more lapses in diabetes management behaviors which in turn contributes to suboptimal glycemic control. This suggestion is similar to the findings in the diabetes family conflict literature, wherein greater family conflict indirectly contributed to poorer glycemic control by decreasing self-care behaviors [7, 29]. From a clinical perspective, widely discordant views on diabetes-related issues between parents and youth may signal to the provider that interventions promoting adaptive communication 
and conflict resolution skills may be of particular benefit $[10,30]$. Annual administration of a diabetes communication survey may also help providers screen for and track parent-youth communication discrepancies to help determine if additional, more intensive intervention is needed.

This study also found no effect of parentand youth-reported frequency of communication on glycemic control and adherence. One possible explanation is that although raw frequency of communication may reflect one aspect of parental involvement, it is necessary to know whether that involvement tends to be positive or negative [14]. For example, some youth perceive their parents' frequent communication as "nagging" [31], while others perceive their parents, specifically fathers, to be under-involved, despite efforts to include them in treatment discussions [32]. Consistent with these findings, results from the present study indicate that frequency of communication generally did not correlate with the tone of communication, with the exception of one item (discussed missed insulin boluses), where greater communication about missed boluses was associated with more negative tone (see Table 1). In cases of mismatched parent-youth communication, research shows increased risk of suboptimal diabetes management $[16,33]$.

It is also plausible that frequency of communication is differentially related to parameters of glycemic control depending on individual-specific diabetes management behaviors. As an additional exploratory items analysis to test this hypothesis, we performed separate regressions to test if individual FCom items significantly predicted $\mathrm{HbA1c}$, after controlling for youth age, duration of diabetes, and gender (see Table 1). When examining FCom items, results indicated that more parent- and youth-reported communication about hypoglycemia (e.g., discussed particular low blood sugar; discussed frequency/number of low blood sugars) corresponded to lower HbA1c values. In contrast, increased communication about hyperglycemia as reported by youth (e.g., discussed missed insulin boluses; discussed frequency/number of high blood sugars) was associated with higher HbA1c. These findings suggest that parents and youth tend to communicate most frequently about issues most relevant to the way in which youth manage their diabetes. That is, when youth maintain tighter glycemic control (and thus are at a higher risk for hypoglycemic events) parent-youth dyads communicate more frequently about low BG values. However, when youth are in suboptimal control, high BG values and insulin dosing are discussed more frequently. If this is the case, increasing the overall frequency of communication is likely inadequate to fully address problem areas in diabetes management without examining all of these self-management factors.

Finally, this study assessed the tone of communication about diabetes management. Consistent with our hypothesis, lower HbA1c values were associated with more youth-perceived positive communication beyond the effects of adherence. However, there was no association between glycemic control and parent-perceived tone or discrepancy between parents and youth on tone of communication. Past research generally supports these findings for youth-reported family communication behaviors, with most studies demonstrating improved glycemic control with increased positive communication behaviors (i.e., support, love, acceptance, warmth) $[15,17,34,35]$, and poorer glycemic control with more youth-reported negative communication styles (i.e., anger, interruption, unsupportiveness) $[17,22,36]$. There is some evidence that mother-reported positive communication behaviors are associated with better glycemic control [35], and negative behaviors are related to worsened control [36], but these findings are inconsistent [34, 35]. No identified research has examined the relationship between discrepancies in tone of communication and glycemic control. The non-significant finding in this study may be due to the limited power to explore discrepancies in parent-youth dyads for tone, but future research will need to establish if this is the case.

Although the results of this study are informative, there are several methodological limitations. As a result of the anonymity of the survey, we were unable to identify whether the mother or father participated, limiting our 
ability to draw more specific conclusions about differences in communication. We also lack information regarding other demographic characteristics of the participants and how many declined the survey, limiting generalizability of the findings. Additionally, the questionnaires selected for this study were limited to promote efficiency and to reduce participant burden. Consequently, additional relevant communication concepts, such as parental monitoring [37, 38], and family responsibility [26] were not assessed. While the CSM and FCI had thus not previously been validated, we took multiple steps to maintain rigor in their formulation and assessment, described below. The CSM assessed diabetes care behaviors and, as seen in other validated behavior scales, exhibited acceptable reliability and correlated significantly with HbA1c levels [17]. The FCI assessed concepts for the most part not covered by other tools but was formulated by consulting other family diabetes scales to inform the manner of assessment items. We noted a high correlation between TCom and a previously validated tool, the Family Conflict Scale [11]. We further evaluated Cronbach alpha scores for the FCom scale, which appeared to measure a single factor with high internal reliability. Nevertheless, the psychometric properties of the TCom scale could not be fully evaluated given that parents and youth did not report communicating about certain diabetes management behaviors within the designated time frame (i.e., 1 week), because of questions regarding tone of communication were not relevant to families not reporting recent communication, only $33 \%$ of participants having data from all questions regarding the tone of communication-rendering inadequate power for Cronbach alpha analyses. Future iterations of the inventory should consider larger sample sizes or assessing tone/frequency over a longer time period (i.e., 1 month) or implementing a more general measure of tone for each item (i.e., "when you talk about this topic, is communication positive or negative?") and performing further validation against other external outcomes or-in the absence of similar tools-with direct observational data.

\section{CONCLUSION}

In spite of the limitations, this preliminary study highlights the complexity of communication in diabetes management and challenges the overly broad notion that more communication leads to improved diabetes control. In fact, in the present study, valence of communication as measured by tone and discrepancies in parent and youth perceptions about how often they are communicating about diabetes management tasks had higher correlations with overall glycemic control than frequency of communication, suggesting that these areas warrant further investigation. In addition, future larger-scale studies are needed to further examine the association between parent-youth discrepancies in tone of communication and glycemic control.

\section{ACKNOWLEDGEMENTS}

The U.S. National Library of Medicine 1R01LM012090 (MDD, RV, DRC, SV) funded the study and contributed toward article processing charges. The remainder of article processing charges were paid for by the authors. All named authors meet the International Committee of Medical Journal Editors (ICMJE) criteria for authorship for this manuscript, take responsibility for the integrity of the work as a whole, and have given final approval to the version to be published. We would also like to acknowledge the support of Jackie Shepard, Jesse Grabman, and Linda Gonder-Frederick for their invaluable assistance in the assessment of these tools.

Disclosures. Mark D. DeBoer, Rupa Valdez, Daniel R. Chernavvsky, Monica Grover, Christine Burt Solorzano, Kirabo Herbert and Stephen Patel have nothing to disclose.

Compliance with Ethics Guidelines. All procedures followed were in accordance with the ethical standards of the responsible committee on human experimentation (institutional and national) and with the Helsinki 
Declaration of 1964, as revised in 2013. Informed consent was obtained from all patients for being included in the study.

Data Availability. The datasets during and/ or analyzed during the current study are available from the corresponding author on reasonable request.

Open Access. This article is distributed under the terms of the Creative Commons Attribution-NonCommercial 4.0 International License (http://creativecommons.org/licenses/ by-nc/4.0/), which permits any noncommercial use, distribution, and reproduction in any medium, provided you give appropriate credit to the original author(s) and the source, provide a link to the Creative Commons license, and indicate if changes were made.

\section{REFERENCES}

1. Gerstl EM, Rabl W, Rosenbauer J, et al. Metabolic control as reflectet by HbA1c in children, adolescents and young adults with type- 1 diabetes mellitus: combined longitudinal analysis including 27,035 patients from 207 centers in Germany and Austria during the last decade. Eur J Pediatr. 2008; 167:447-53.

2. Hanna KM, Juarez B, Lenss SS, Guthrie D. Parent-adolescent communication and support for diabetes management as reported by adolescents with type 1 diabetes. Issues Compr Pediatr Nurs. 2003;26:145-58.

3. Wysocki T, Meinhold P, Cox DJ, Clarke WL. Survey of diabetes professionals regarding developmental changes in diabetes self-care. Diabetes Care. 1990;13:65-8.

4. Sawyer SM, Aroni RA. Self-management in adolescents with chronic illness. What does it mean and how can it be achieved? Med J Aust. 2005;183:405-9.

5. Guilfoyle SM, Crimmins NA, Hood KK. Blood glucose monitoring and glycemic control in adolescents with type 1 diabetes: meter downloads versus self-report. Pediatr Diabetes. 2011;12:560-6.

6. Campbell M, Schatz D, Chen V, et al. A contrast between children and adolescents with excellent and poor control: the T1D exchange clinic registry experience. Pediatr Diabetes. 2014;15:110-7.

7. Hilliard ME, Wu YP, Rausch J, Dolan LM, Hood KK. Predictors of deteriorations in diabetes management and control in adolescents with type 1 diabetes. J Adolesc Health. 2013;52:28-34.

8. Olinder AL, Kernell A, Smide B. Missed bolus doses: devastating for metabolic control in CSII-treated adolescents with type 1 diabetes. Pediatr Diabetes. 2009;10:142-8.

9. VanderWel BW, Messer LH, Horton LA, et al. Missed insulin boluses for snacks in youth with type 1 diabetes. Diabetes Care. 2010;33:507-8.

10. Laffel LMB, Vangsness L, Connell A, Goebel-Fabbri A, Butler D, Anderson BJ. Impact of ambulatory, family-focused teamwork intervention on glycemic control in youth with type 1 diabetes. J Pediatr. 2003;142:409-16.

11. Anderson BJ, Vangsness L, Connell A, Butler D, Goebel-Fabbri A, Laffel LM. Family conflict, adherence, and glycaemic control in youth with short duration type 1 diabetes. Diabet Med. 2002;19:635-42.

12. Vesco AT, Anderson BJ, Laffel LM, Dolan LM, Ingerski LM, Hood KK. Responsibility sharing between adolescents with type 1 diabetes and their caregivers: importance of adolescent perceptions on diabetes management and control. J Pediatr Psychol. 2010;35:1168-77.

13. Ellis DA, Podolski CL, Frey M, Naar-King S, Wang B, Moltz K. The role of parental monitoring in adolescent health outcomes: impact on regimen adherence in youth with type 1 diabetes. J Pediatr Psychol. 2007;32:907-17.

14. Wysocki T, Taylor A, Hough BS, Linscheid TR, Yeates KO, Naglieri JA. Deviation from developmentally appropriate self-care autonomy. Association with diabetes outcomes. Diabetes Care. 1996;19:119-25.

15. Berg CA, King PS, Butler JM, Pham P, Palmer D, Wiebe DJ. Parental involvement and adolescents' diabetes management: the mediating role of self-efficacy and externalizing and internalizing behaviors. J Pediatr Psychol. 2011;36:329-39.

16. Cameron FJ, Skinner TC, de Beaufort CE, et al. Are family factors universally related to metabolic outcomes in adolescents with type 1 diabetes? Diabet Med. 2008;25:463-8.

17. Lewin $A B$, Heidgerken AD, Geffken GR, et al. The relation between family factors and metabolic 
control: the role of diabetes adherence. J Pediatr Psychol. 2006;31:174-83.

18. Drotar D, Ittenbach R, Rohan JM, Gupta R, Pendley JS, Delamater A. Diabetes management and glycemic control in youth with type 1 diabetes: test of a predictive model. J Behav Med. 2013;36:234-45.

19. Hood KK, Butler DA, Anderson BJ, Laffel LM. Updated and revised diabetes family conflict scale. Diabetes Care. 2007;30:1764-9.

20. Miller-Johnson S, Emery RE, Marvin RS, Clarke W, Lovinger R, Martin M. Parent-child relationships and the management of insulin-dependent diabetes mellitus. J Consult Clin Psychol. 1994;62:603-10.

21. Geffken GR, Lehmkuhl H, Walker KN, et al. Family functioning processes and diabetic ketoacidosis in youths with type I diabetes. Rehabil Psychol. 2008;53:231-7.

22. Duke DC, Geffken GR, Lewin AB, Williams LB, Storch EA, Silverstein JH. Glycemic control in youth with type 1 diabetes: family predictors and mediators. J Pediatr Psychol. 2008;33:719-27.

23. Martin MT, Miller-Jonson S, Kitzmann KM, Emery RE. Parent-child relationships and insulin-dependent diabetes mellitus: observational ratings of clinically relevant dimensions. J Fam Psychol. 1998;12:102-11.

24. Laffel LM, Connell A, Vangsness L, Goebel-Fabbri A, Mansfield A, Anderson BJ. General quality of life in youth with type 1 diabetes: relationship to patient management and diabetes-specific family conflict. Diabetes Care. 2003;26:3067-73.

25. Weissberg-Benchell J, Nansel T, Holmbeck G, et al. Study SCotFMoD: generic and diabetes-specific parent-child behaviors and quality of life among youth with type 1 diabetes. J Pediatr Psychol. 2009;34:977-88.

26. Anderson BJ, Holmbeck G, Iannotti RJ, et al. Dyadic measures of the parent-child relationship during the transition to adolescence and glycemic control in children with type 1 diabetes. Fam Syst Health. 2009;27:141-52.

27. American Diabetes Association. (11) Children and adolescents. Diabetes Care. 2015;38(Suppl):S70-6.

28. Lewin AB, LaGreca AM, Geffken GR, et al. Validity and reliability of an adolescent and parent rating scale of type 1 diabetes adherence behaviors: the Self-Care Inventory (SCI). J Pediatr Psychol. 2009;34:999-1007.
29. Hilliard ME, Guilfoyle SM, Dolan LM, Hood KK. Prediction of adolescents' glycemic control 1 year after diabetes-specific family conflict: the mediating role of blood glucose monitoring adherence. Arch Pediatr Adolesc Med. 2011;165:624-9.

30. Wysocki T, Harris MA, Buckloh LM, et al. Randomized trial of behavioral family systems therapy for diabetes: maintenance of effects on diabetes outcomes in adolescents. Diabetes Care. 2007;30:555-60.

31. Weinger K, O’Donnell KA, Ritholz MD. Adolescent views of diabetes-related parent conflict and support: a focus group analysis. J Adolesc Health. 2001;29:330-6.

32. Seiffge-Krenke I. "Come on, say something, dad!": communication and coping in fathers of diabetic adolescents. J Pediatr Psychol. 2002;27:439-50.

33. Wiebe DJ, Berg CA, Korbel C, et al. Children's appraisals of maternal involvement in coping with diabetes: enhancing our understanding of adherence, metabolic control, and quality of life across adolescence. J Pediatr Psychol. 2005;30:167-78.

34. Berg CA, Butler JM, Osborn P, et al. Role of parental monitoring in understanding the benefits of parental acceptance on adolescent adherence and metabolic control of type 1 diabetes. Diabetes Care. 2008;31:678-83.

35. Wysocki T, Harris MA, Buckloh LM, et al. Randomized, controlled trial of behavioral family systems therapy for diabetes: maintenance and generalization of effects on parent-adolescent communication. Behav Ther. 2008;39:33-46.

36. Iskander JM, Rohan JM, Pendley JS, Delamater A, Drotar D. A 3-year prospective study of parent-child communication in early adolescents with type 1 diabetes: relationship to adherence and glycemic control. J Pediatr Psychol. 2015;40:109-20.

37. Ellis DA, Templin TN, Podolski CL, Frey MA, Naar-King S, Moltz K. The parental monitoring of diabetes care scale: development, reliability and validity of a scale to evaluate parental supervision of adolescent illness management. J Adolesc Health. 2008;42:146-53.

38. Palmer DL, Osborn P, King PS, et al. The structure of parental involvement and relations to disease management for youth with type 1 diabetes. J Pediatr Psychol. 2011;36:596-605. 